\title{
Power Spectra from an Inflaton Coupled to the Gauss-Bonnet Term
}

\author{
Zong-Kuan Guo* \\ Fakultät für Physik, Universität Bielefeld, Postfach 100131, 33501 Bielefeld, Germany \\ E-mail: guozk@physik.uni-bielefeld.de

\section{Dominik J. Schwarz} \\ Fakultät für Physik, Universität Bielefeld, Postfach 100131, 33501 Bielefeld, Germany \\ E-mail: dschwarz@physik.uni-bielefeld.de
}

\begin{abstract}
We consider power-law inflation with a Gauss-Bonnet correction inspired by string theory. We analyze the stability of cosmological perturbations and obtain the allowed parameter space. We find that for GB-dominated inflation ultra-violet instabilities of either scalar or tensor perturbations show up on small scales. The Gauss-Bonnet correction with a positive (or negative) coupling may lead to a reduction (or enhancement) of the tensor-to-scalar ratio in the potential-dominated case. We place tight constraints on the model parameters by making use of the WMAP 5-year data.
\end{abstract}

International Workshop on Cosmic Structure and Evolution - Cosmology2009,

September 23-25, 2009

Bielefeld, Germany

\footnotetext{
${ }^{*}$ Speaker.
} 


\section{Outline}

- Motivations

- Cosmological perturbations

- Power-law inflation

- Conclusions

\section{Motivations}

- Inflation: Some cosmological puzzles, such as the horizon problem and the flatness problem, can be explained in the inflation scenario. The most important property of inflation is that it can generate irregularities in the Universe, which may lead to the formation of structure. However, so far the nature of the inflaton has been an open question.

- Higher order corrections: It is known that there are correction terms of higher orders in the curvature to the lowest effective supergravity action coming from superstrings. The simplest correction is the Gauss-Bonnet (GB) term.

- Questions: Does the GB term drive acceleration of the Universe? If so, is it possible to generate nearly scale-invariant curvature perturbations? If not, when the GB term is subdominated, what is the influence on the power spectra? How strong WMAP data constrain the GB coupling?

\section{Cosmological perturbations}

Our action:

$$
S=\int d^{4} x \sqrt{g}\left[\frac{1}{2} R-\frac{\omega}{2}(\nabla \phi)^{2}-V(\phi)-\frac{1}{2} \xi(\phi) R_{\mathrm{GB}}^{2}\right],
$$

$R$ : the Ricci scalar, $\phi$ : a scalar field, $V(\phi)$ : a potential, $\omega= \pm 1$ : canonical or phantom-like scalar field, $R_{\mathrm{GB}}^{2}=R_{\mu v \rho \sigma} R^{\mu v \rho \sigma}-4 R_{\mu v} R^{\mu v}+R^{2}$ : the GB term, $\xi(\phi)$ : the GB coupling.

Background equations: In a spatially-flat Friedmann-Robertson-Walker Universe, the background equations read

$$
\begin{aligned}
& 6 H^{2}=\omega \dot{\phi}^{2}+2 V+24 \dot{\xi} H^{3}, \\
& 2 \dot{H}=-\omega \dot{\phi}^{2}+4 \ddot{\xi} H^{2}+4 \dot{\xi} H\left(2 \dot{H}-H^{2}\right) .
\end{aligned}
$$

To compare the contributions from the potential and the GB term, we use the ratio of the second to the third term on the right-hand side of the Friedmann equation,

$$
\lambda=\frac{V}{12 \dot{\xi} H^{3}} .
$$

$|\lambda|>1$ : a potential-dominated model, $|\lambda|<1$ : a GB-dominated model. 
Scalar perturbation equations:

$$
\begin{gathered}
v^{\prime \prime}+\left(c_{\mathscr{R}}^{2} k^{2}-\frac{z_{\mathscr{R}}^{\prime \prime}}{z_{\mathscr{R}}}\right) v=0, \\
z_{\mathscr{R}}^{2}=\frac{a^{2}\left(\omega \dot{\phi}^{2}-12 I \dot{\xi} H^{2}\right)}{(H+I)^{2}}, \\
c_{\mathscr{R}}^{2}=1+\frac{-16 I \dot{\xi} \dot{H}+8 I^{2}(\ddot{\xi}-\dot{\xi} H)}{\omega \dot{\phi}^{2}-12 I \dot{\xi} H^{2}},
\end{gathered}
$$

with $I=-2 \dot{\xi} H^{2} /(1-4 \dot{\xi} H)$.

Assuming: a) $z_{\mathscr{R}}=Q_{\mathscr{R}}(-\tau)^{1 / 2-v_{\mathscr{R}}}$ with $Q_{\mathscr{R}}$ and $v_{\mathscr{R}}$ constant. b) $c_{\mathscr{R}}^{2}$ is a positive constant. The general solution is a linear combination of Hankel functions

$$
v=e^{i\left(2 v_{\mathscr{R}}+1\right) \pi / 4} \frac{\sqrt{-\pi \tau}}{2}\left[c_{1} H_{v_{\mathscr{R}}}^{(1)}\left(-c_{\mathscr{R}} k \tau\right)+c_{2} H_{v_{\mathscr{R}}}^{(2)}\left(-c_{\mathscr{R}} k \tau\right)\right] .
$$

Choose $c_{1}=1$ and $c_{2}=0$, so that

$$
v \rightarrow \frac{1}{\sqrt{2 c_{\mathscr{R}} k}} e^{-i c_{\mathscr{R}} k \tau} \quad \text { as } c_{\mathscr{R}} k \tau \rightarrow-\infty .
$$

for long wavelength perturbations $\left(c_{\mathscr{R}} k|\tau| \ll 1\right)$

$$
v \rightarrow e^{i\left(2 v_{\mathscr{R}}-1\right) \pi / 4} \frac{(-\tau)^{1 / 2-v_{\mathscr{R}}} c_{\mathscr{R}}^{-v_{\mathscr{R}}}}{4} \frac{\Gamma\left(v_{\mathscr{R}}\right)}{\Gamma(3 / 2)}\left(\frac{k}{2}\right)^{-v_{\mathscr{R}}} .
$$

The power spectrum of curvature perturbations

$$
\mathscr{P}_{\mathscr{R}}=\frac{k^{3}}{2 \pi^{2}}\left|\frac{v}{z_{\mathscr{R}}}\right|^{2}=\frac{c_{\mathscr{R}}^{-2 v_{\mathscr{R}}}}{4 \pi^{2} Q_{\mathscr{R}}^{2}}\left(\frac{\Gamma\left(v_{\mathscr{R}}\right)}{\Gamma(3 / 2)}\right)^{2}\left(\frac{k}{2}\right)^{3-2 v_{\mathscr{R}}},
$$

with spectral index

$$
n_{\mathscr{R}}-1=3-2 v_{\mathscr{R}}
$$

Tensor perturbation equations:

$$
\begin{gathered}
u^{\prime \prime}+\left(c_{T}^{2} k^{2}-\frac{z_{T}^{\prime \prime}}{z_{T}}\right) u=0, \\
z_{T}^{2}=a^{2}(1-4 \dot{\xi} H), \\
c_{T}^{2}=1-\frac{4(\ddot{\xi}-\dot{\xi} H)}{1-4 \dot{\xi} H} .
\end{gathered}
$$

Assuming: a) $z_{T}=Q_{T}(-\tau)^{1 / 2-v_{T}}$ with $Q_{T}$ and $v_{T}$ constant. b) $c_{T}^{2}$ is a positive constant. The 
power spectrum of tensor perturbations

$$
\mathscr{P}_{T}=\frac{2 k^{3}}{2 \pi^{2}}\left|\frac{2 u}{z_{T}}\right|^{2}=\frac{8 c_{T}^{-2 v_{T}}}{4 \pi^{2} Q_{T}^{2}}\left(\frac{\Gamma\left(v_{T}\right)}{\Gamma(3 / 2)}\right)^{2}\left(\frac{k}{2}\right)^{3-2 v_{T}} .
$$

The spectral index

$$
n_{T}=3-2 v_{T}
$$

The tensor-to-scalar ratio

$$
r \equiv \frac{\mathscr{P}_{T}}{\mathscr{P}_{\mathscr{R}}}=8 \frac{Q_{\mathscr{R}}^{2}}{Q_{T}^{2}} \frac{c_{\mathscr{R}}^{2 v_{\mathscr{R}}}}{c_{T}^{2 v_{T}}}\left(\frac{\Gamma\left(v_{T}\right)}{\Gamma\left(v_{\mathscr{R}}\right)}\right)^{2}\left(\frac{k}{2}\right)^{2 v_{\mathscr{R}}-2 v_{T}} .
$$

\section{Power-law inflation}

Power-law solution:

$$
a \propto t^{1 / \gamma}, \quad H=\frac{1}{\gamma t} .
$$

The potential and the coupling take the form

$$
\dot{\xi}=\alpha t, \quad V=\frac{\beta}{\gamma t^{2}},
$$

Note: $\gamma$, a positive constant; $\alpha$, a constant; $\beta$, a positive constant.

$$
(\beta+1) \gamma^{2}-(2 \alpha+3) \gamma+10 \alpha=0,
$$

which has two solutions, $\gamma_{+}$and $\gamma_{-}$,

$$
\gamma_{ \pm}=\frac{(2 \alpha+3) \pm \sqrt{(2 \alpha+3)^{2}-40 \alpha(\beta+1)}}{2(\beta+1)} .
$$

In this case the potential-to-GB ratio, the scalar field, the couple and the potential can be written as

$$
\begin{aligned}
& \lambda=\frac{\gamma^{2} \beta}{12 \alpha}, \\
& \phi=\frac{1}{\gamma} \sqrt{\frac{2\left|\gamma^{2}-2 \alpha \gamma-2 \alpha\right|}{\gamma} \ln t} \\
& \xi(\phi)=\frac{\alpha}{2} \exp \left(\sqrt{\frac{2 \gamma}{\left|\gamma^{2}-2 \alpha \gamma-2 \alpha\right|}} \gamma \phi\right), \\
& V(\phi)=\frac{\beta}{\gamma} \exp \left(-\sqrt{\frac{2 \gamma}{\left|\gamma^{2}-2 \alpha \gamma-2 \alpha\right|}} \gamma \phi\right) .
\end{aligned}
$$

Note: the sign of $\omega$ depends on the sign of $\left(\gamma^{2}-2 \alpha \gamma-2 \alpha\right)$; take the positive sign of $\phi$; both the potential and the coupling take the exponential form. 
Power spectra:

$$
\begin{aligned}
& c_{\mathscr{R}}^{2}=1+\frac{16 \alpha^{2}\left(-\gamma^{2}+5 \alpha \gamma-\alpha\right)}{\left[\left(\gamma^{2}-2 \alpha \gamma-2 \alpha\right)(\gamma-4 \alpha)+12 \alpha^{2}\right](\gamma-4 \alpha)}, \\
& n_{\mathscr{R}}-1=3-\left|\frac{3-\gamma}{1-\gamma}\right|, \\
& c_{T}^{2}=1-\frac{4 \alpha(\gamma-1)}{\gamma-4 \alpha}, \\
& n_{T}=3-\left|\frac{3-\gamma}{1-\gamma}\right|, \\
& r=16 \frac{\left(\gamma^{2}-2 \alpha \gamma-2 \alpha\right)(\gamma-4 \alpha)+12 \alpha^{2}}{(\gamma-6 \alpha)^{2}}\left(\frac{c_{\mathscr{R}}^{2}}{c_{T}^{2}}\right)^{v_{\mathscr{R}}} .
\end{aligned}
$$

Note: a red spectrum for $0<\gamma<1 ; r$ is independent of $k$.

Two limiting cases:

(1) A potential-driven inflation, i.e., $\alpha=0$,

$$
\begin{aligned}
& \omega=1, \quad \gamma_{-}=0, \quad \gamma=\gamma_{+}, \\
& 0<\gamma<1 \Longrightarrow \beta>2, \\
& c_{\mathscr{R}}^{2}=c_{T}^{2}=1, \\
& n_{\mathscr{R}}-1=n_{T} \approx-2 \gamma(\gamma \ll 1), \quad r=-8 n_{T} .
\end{aligned}
$$

(2) A GB-driven inflation, i.e., $\beta=0$,

$$
\begin{aligned}
& \omega=-1, \quad \gamma_{+}>1, \quad \gamma=\gamma_{-}, \\
& 0<\gamma<1 \Longrightarrow \alpha<1 / 4, \\
& c_{\mathscr{R}}^{2}>0, \quad c_{T}^{2}=2 \gamma-5<0 \Longrightarrow \text { instability. }
\end{aligned}
$$

\section{Conclusions}

- For GB-dominated inflation ultra-violet instabilities of either scalar or tensor perturbations show up on small scales.

- The Gauss-Bonnet correction with a positive (or negative) coupling may lead to a reduction (or enhancement) of the tensor-to-scalar ratio in the potential-dominated case.

- We place tight constraints on the GB coupling by making use of the WMAP 5-year data.

$$
-1 \times 10^{-4} \lesssim \alpha \lesssim 4 \times 10^{-4} \quad(95 \% \mathrm{CL})
$$




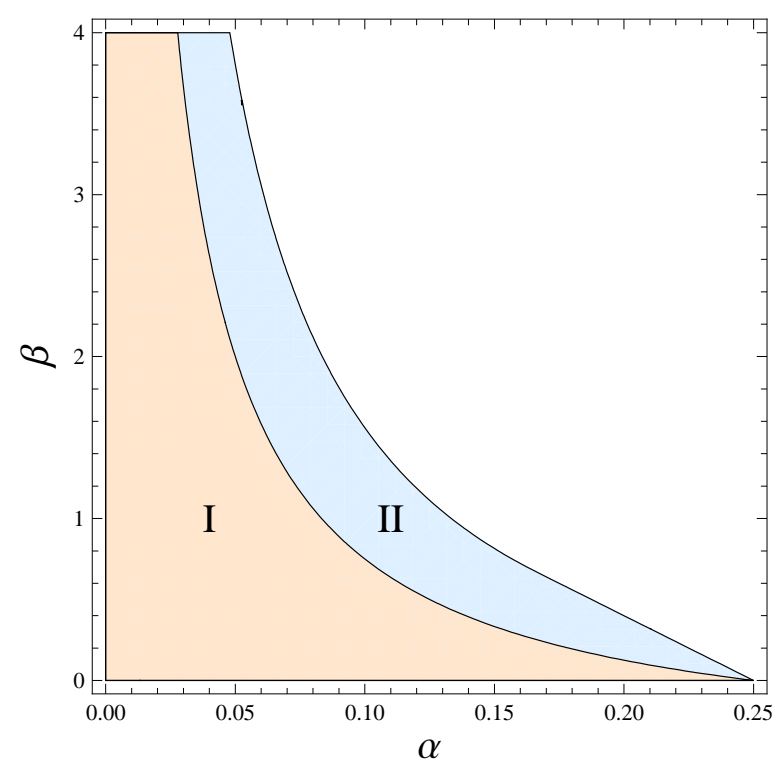

Figure 1: Parameter space $(\alpha, \beta)$ for inflationary solutions with $0<\gamma_{-}<1$. In region I, tensor modes are unstable on small scales, while scalar modes are stable. The opposite holds in region II.

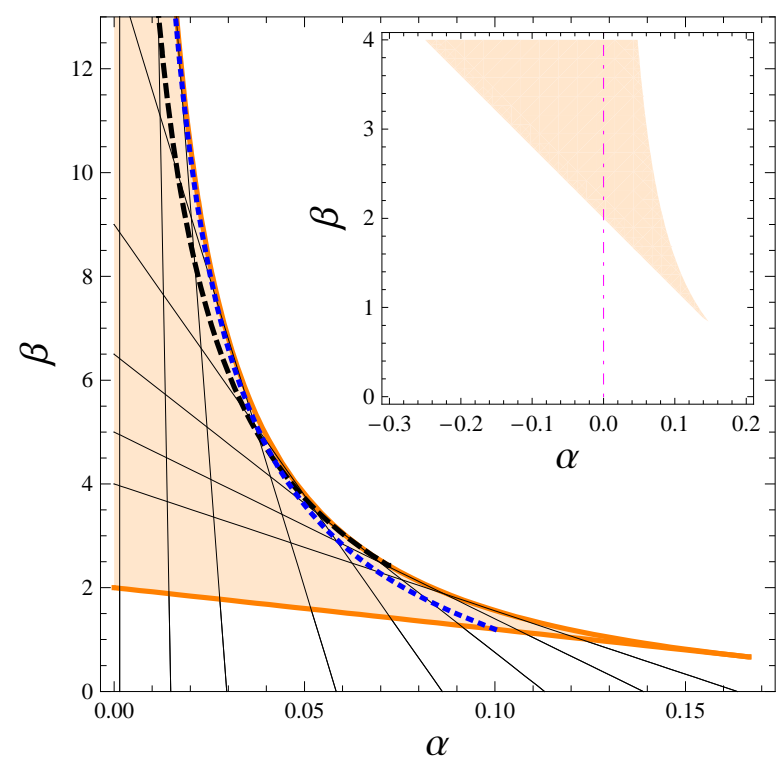

Figure 2: Parameter space $(\alpha, \beta)$ for inflationary solutions with $0<\gamma_{+}<1$. In the shaded region both scalar and tensor modes are stable on small scales. Solid lines correspond to $\gamma=0.005,0.05,0.1,0.2,0.3,0.4$, 0.5, 0.6 (from bottom left to right). Models with $\omega=-1(\omega=1)$ are above (below) the black dashed line. The blue dotted line represents the potential-GB equality $(\lambda=1)$. The potential-dominated (GB-dominated) models are below (above) this line. 


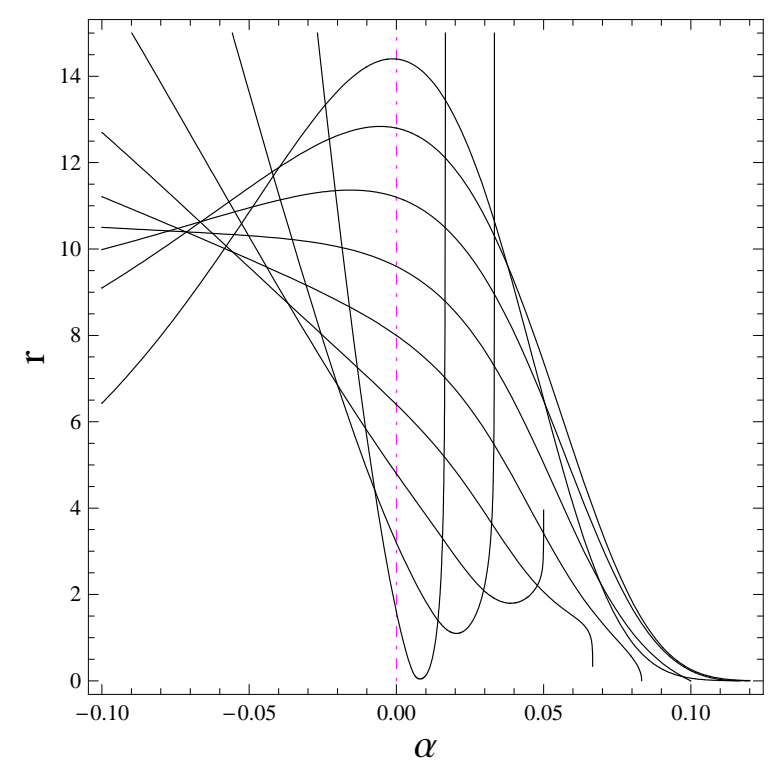

Figure 3: Tensor-to-scalar ratio $r$ versus GB-coupling parameter $\alpha$ for inflationary solutions with $\gamma=0.1$, $0.2,0.3,0.4,0.5,0.6,0.7,0.8,0.9$ (from bottom to top along the dash-dotted line).

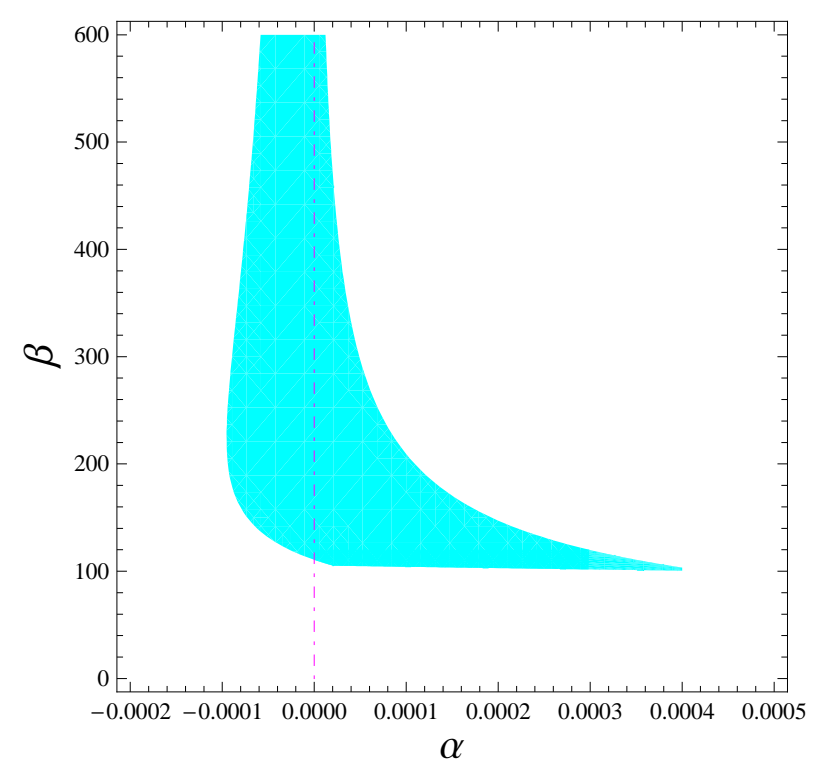

Figure 4: Observational constraints on the inflation-model parameters from the WMAP 5-year analysis. In the shaded region, $0.942<n_{\mathscr{R}}<1$ and $0<r<0.43$. 